\title{
The Triform algorithm: improved sensitivity and specificity in ChIP-Seq peak finding
}

\author{
Karl Kornacker ${ }^{1}$, Morten Beck Rye ${ }^{2}$, Tony Håndstad ${ }^{2}$ and Finn Drabløs ${ }^{2^{*}}$
}

\begin{abstract}
Background: Chromatin immunoprecipitation combined with high-throughput sequencing (ChIP-Seq) is the most frequently used method to identify the binding sites of transcription factors. Active binding sites can be seen as peaks in enrichment profiles when the sequencing reads are mapped to a reference genome. However, the profiles are normally noisy, making it challenging to identify all significantly enriched regions in a reliable way and with an acceptable false discovery rate.

Results: We present the Triform algorithm, an improved approach to automatic peak finding in ChIP-Seq enrichment profiles for transcription factors. The method uses model-free statistics to identify peak-like distributions of sequencing reads, taking advantage of improved peak definition in combination with known characteristics of ChIP-Seq data.

Conclusions: Triform outperforms several existing methods in the identification of representative peak profiles in curated benchmark data sets. We also show that Triform in many cases is able to identify peaks that are more consistent with biological function, compared with other methods. Finally, we show that Triform can be used to generate novel information on transcription factor binding in repeat regions, which represents a particular challenge in many ChIP-Seq experiments. The Triform algorithm has been implemented in $\mathrm{R}$, and is available via http://tare.medisin.ntnu.no/triform.
\end{abstract}

Keywords: ChIP-Seq, Peak finding, Benchmark, Repeats

\section{Background}

Chromatin immunoprecipitation combined with highthroughput sequencing (ChIP-Seq) is currently the method of choice for genome-wide mapping of binding sites for transcription factors (TFs) on DNA [1-3]. This is achieved by using DNA fragmentation after DNAbinding proteins have been cross-linked to the genome. The fragmentation is followed by selection of fragments bound by the TF of interest, using an antibody targeting this factor. The selected genomic fragments are then released, sequenced, and mapped to a reference genome. There, the genomic locations bound by the TF will be enriched with matching sequencing reads.

\footnotetext{
* Correspondence: finn.drablos@ntnu.no

${ }^{2}$ Department of Cancer Research and Molecular Medicine, Norwegian University of Science and Technology (NTNU), P.O. Box 8905, NO-7491 Trondheim, Norway

Full list of author information is available at the end of the article
}

An essential step in the analysis of ChIP-Seq data is the genome-wide identification of enriched regions. Several software tools have been developed to perform this task [4,5], but benchmarking has demonstrated that there is room for improvement in the existing approaches [6]. There are alternative definitions of what constitutes a peak, and useful characteristics of ChIPSeq profiles may not be fully utilized. A typical example is the shift property [7], which occurs because the full sequence fragments, typically with an average length around $200 \mathrm{bp}$, are sequenced for only $25-50 \mathrm{bp}$ from each side. Other examples are the use of independent control samples [8], separation of overlapping enrichment profiles $[9,10]$, or optimal use of statistical approaches to separate signal from noise [11]. Our conclusion in a previous study [6] was that different programs focus on different characteristics of the ChIP-Seq data, but that no program takes all the characteristics into account. Though several programs achieve a high

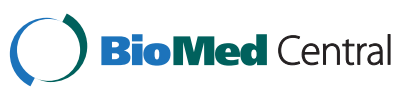


coverage of true enrichment profiles, the trade-off has often been an intolerably high false discovery rate (FDR).

A major limitation in the development of improved approaches has been the lack of proper benchmarks to compare the performance of different methods [12]. Because of this, different strategies for performance evaluation have been used, the most common being motif occurrences in the proximity of the ChIP-Seq peaks $[4,5,8,13]$, and overlap with experimentally confirmed qPCR sites [4,5]. However, both evaluation methods have important limitations $[6,12]$, and when looking at a limited number of binding regions, the preferred evaluation method remains visual assessment of local enrichment profiles in a genome browser. To compensate for the lack of benchmarks in ChIP-Seq analysis, we have previously manually curated a benchmark data set based on visual inspection of ChIP-Seq profiles from three different TFs: NRSF (also known as REST), SRF, and MAX [6]. Individual regions were visually assessed relative to relevant criteria, including peak-like shape, peak shift, lack of signal in control sample, and motif occurrence in peak region, and the regions were classified either as real peaks or as noise or artifacts. The idea was that such a benchmark can be used to evaluate and improve both new and existing efforts for the automatic analysis of ChIP-Seq data for TFs.

In this study, we present an improved approach for automatic identification of peaks in ChIP-Seq enrichment profiles, called the Triform method. Triform uses robust genome-wide statistical tests to detect three different forms of peak-like enrichment profiles, taking advantage of the profile characteristics mentioned above. Overfitting is precluded by the fact that Triform is based on model-free statistical tests and uses a minimal number of preset parameters based on the general properties of the ChIP-Seq data sets. The Triform algorithm is model free in the sense that it relies on the Hoel test [14] which is fully nonparametric, i.e. free from any assumed relationships or fitted parameters. In particular, the Hoel test is free from any assumed background model and is therefore more robust than model-based tests, which depend on locally uniform background models and fitted background parameters. When evaluated on the benchmark data set of peak profiles, Triform outperformed both newly developed and previously evaluated programs for the automatic detection of enrichment profiles, for all three TFs studied. The good performance of Triform was further confirmed using tests on motif enrichment in significant peak regions.

Since TFs often co-regulate genes that are involved in specific biological processes, we would expect such processes to be overrepresented in the annotation of genes associated with regions for TF binding from the
ChIP-Seq experiment. We therefore used statistical overrepresentation analysis on peak sets from the main peak-finding programs that are compared here, and showed that peak sets from Triform in most cases give the most significant overrepresentation of relevant annotation terms.

To illustrate the potential of improved peak finding to generate novel information, we further analyzed the DNA sequence motifs identified de novo within the Triform SRF peak regions. In addition to the canonical SRF motif, we discovered a significant co-occurrence of SRF, ELK1, and NFY motifs within LTR/ERV1/MER57 repeats, and these particular repeats were significantly co-located with genes associated with cancer. This exemplifies how optimal identification of peak regions may generate novel information.

\section{Results and discussion}

\section{The Triform algorithm}

The main problem with peak finding in ChIP-Seq data for TFs is the reliable differentiation between peaks and noise. Many algorithms define a peak as a region of significantly elevated coverage of sequencing reads. Consequently, such algorithms tend to accept false positives in the form of noisy plateaus, i.e., wide regions of elevated coverage lacking a distinctive core sub-region and lacking a well-defined shift between coverage profiles on opposite strands. Such regions are expected to be present, to some extent, in any ChIP-Seq data set.

The Triform algorithm defines a peak as a region with a significantly negative mean second derivative of the coverage profile, using model-free test statistics developed by Hoel [14]. Such regions have inherently limited width, peak sub-regions are directly identified, and these sub-regions can be used to test for well-defined shifts between overlapping profiles on opposite strands. The test can also handle overlapping peaks. Consequently, the Triform algorithm is able to reject false positive noisy plateaus, thereby increasing specificity with little or no loss of sensitivity. This is used in combination with other important features and data, in particular control data and biological replicates. Additional sequencing of control data emulating tag enrichment profiles created without targeting any specific TF has become standard in ChIP-Seq, and may be used to improve the separation of true peaks from noise and artificial enrichment. The same is true for biological replicates.

\section{Calculation of model-free test statistics for local peak-like forms on each strand}

At each strand location $x$ the raw ChIP-Seq coverage $C(x)$ is formally regarded as a Poisson variate with parameter $\lambda(x)=E[C(x)]$. The formal Poisson model leaves 
the Poisson parameter unspecified and does not assume homogeneity across technical replicates, but does imply that any summation of independently measured Poisson variates yields another Poisson variate.

For read width $w$ and fixed parameter $\delta>w$ (see Table 1$)$, the three coverage values $\{C(x-\delta), C(x)$, $C(x+\delta)\}$ at any location $x$ are formally regarded as independently measured Poisson variates with parameters $\{\lambda(x-\delta), \lambda(x), \lambda(x+\delta)\}$.

The following three alternative hypotheses are tested for local peak-like forms (see Figure 1a):

$$
\begin{aligned}
& 2 \lambda(x)>\lambda(x-\delta)+\lambda(x+\delta) \\
& \lambda(x)>\lambda(x-\delta) \\
& \lambda(x)>\lambda(x+\delta)
\end{aligned}
$$

The above three hypothesized peak-like forms will be annotated as Form 1, Form 2, and Form 3 peaks, respectively. In Form 1 peaks, the peak coverage is higher than the average flanking coverage, while in Form 2 and Form 3 peaks the peak coverage is higher than the leftor right-flanking coverage, respectively (Figure 1a).

The following three model-free test statistics for peaklike forms are based on equation (11) of Hoel [14]. These statistics have approximately standard normal distributions even for arbitrarily low positive total counts.

$$
\begin{aligned}
& z 1(x ; \delta)=\frac{2 C(x)-(C(x-\delta)+C(x+\delta))}{\sqrt{2(C(x-\delta)+C(x)+C(x+\delta))}} \\
& z 2(x ; \delta)=\frac{C(x)-C(x-\delta)}{\sqrt{C(x-\delta)+C(x)}} \\
& z 3(x ; \delta)=\frac{C(x)-C(x+\delta)}{\sqrt{C(x+\delta)+C(x)}}
\end{aligned}
$$

\section{Calculation of model-free test statistics for local enrichment on each strand}

At each strand location $x$ the raw coverage $B(x)$ of the control sample is formally regarded as a Poisson variate with Poisson parameter $\mu(x)=E[B(x)]$. For a given ratio $r$ between the control and ChIP-Seq library sizes (see Table 1), the tested hypothesis of local enrichment is:

$$
r \cdot \lambda(x)>\mu(x)
$$

The model-free test statistic for local enrichment, $z 4(x)$, is calculated according to (8). The local enrichment ratio, $E R(x)$, is calculated according to (9).

$$
\begin{aligned}
& z 4(x)=\frac{r \cdot C(x)-B(x)}{\sqrt{r(B(x)+C(x))}} \\
& E R(x)=\frac{1+r \cdot C(x)}{1+B(x)}
\end{aligned}
$$

\section{Calculation of local inter-strand lags between overlapping peaks on opposite strands}

Figure 1c illustrates the range of locations involved in the calculation of the local inter-strand lag between two overlapping Form 1 peak regions on opposite strands. The range starts at the $($ start $-\delta)$ position on the forward strand and ends at the $(e n d+\delta)$ position on the reverse strand. The optimal lag is taken as the one that maximizes the cross-correlation between the forward and reverse coverage distributions within the specified range.

\section{Procedure for detecting local peak-like forms}

Parameters and default parameter settings are shown in Table 1 . The parameters are fixed values to avoid overfitting. The default values have been chosen to reflect well-known properties of ChIP-Seq data. For example, the default value of the spacing parameter $\delta$ is $150 \mathrm{bp}$, which is comparable to the length of DNA around a nucleosome. The cut-off for the minimum local enrichment ratio min.er is needed because non-specific ChIP-Seq coverage is often significantly higher than the coverage in the control sample. Currently the min.er cut-off value is calculated for each strand as the $3 / 8$ quantile of the enrichment ratios in significantly enriched Form 1 peaks. The choice of quantile cut-off value does not seem to be critical because the $1 / 4$ and $1 / 2$ quantiles were nearly equal in all tested data sets.

Table 1 Triform parameters

\begin{tabular}{ll}
\hline Parameter & Description \\
\hline$\delta$ & read width, symmetrically extended to a fixed value (default 100 bp) \\
\hline$r$ & fixed spacing between central and flanking locations (must be $>$ w, default 150 bp) \\
\hline min.z & actual ratio between control and ChIP-Seq library sizes \\
\hline min.n & fixed minimum upper-tail z-value (default corresponds to standard normal $p=0.1)$ \\
\hline min.lag & fixed minimum number of bp (peak width) in peak-like region (default 10 bp) \\
\hline
\end{tabular}




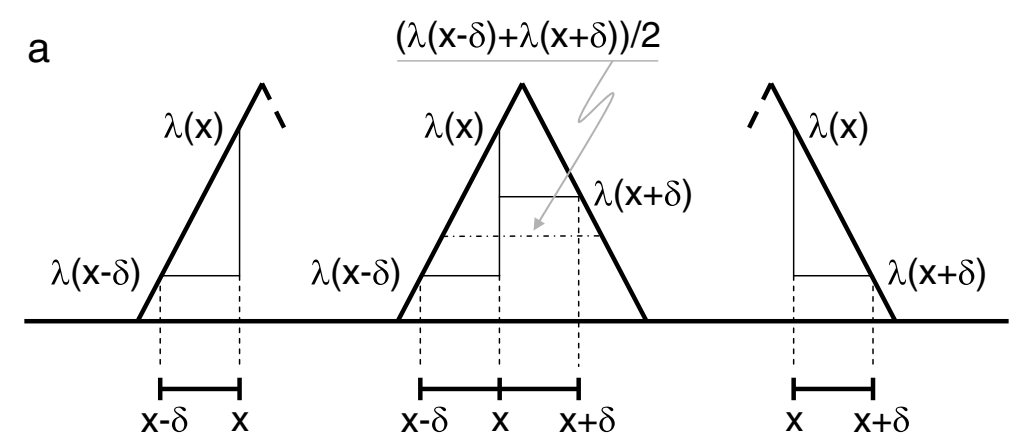

Form 2

Form 1

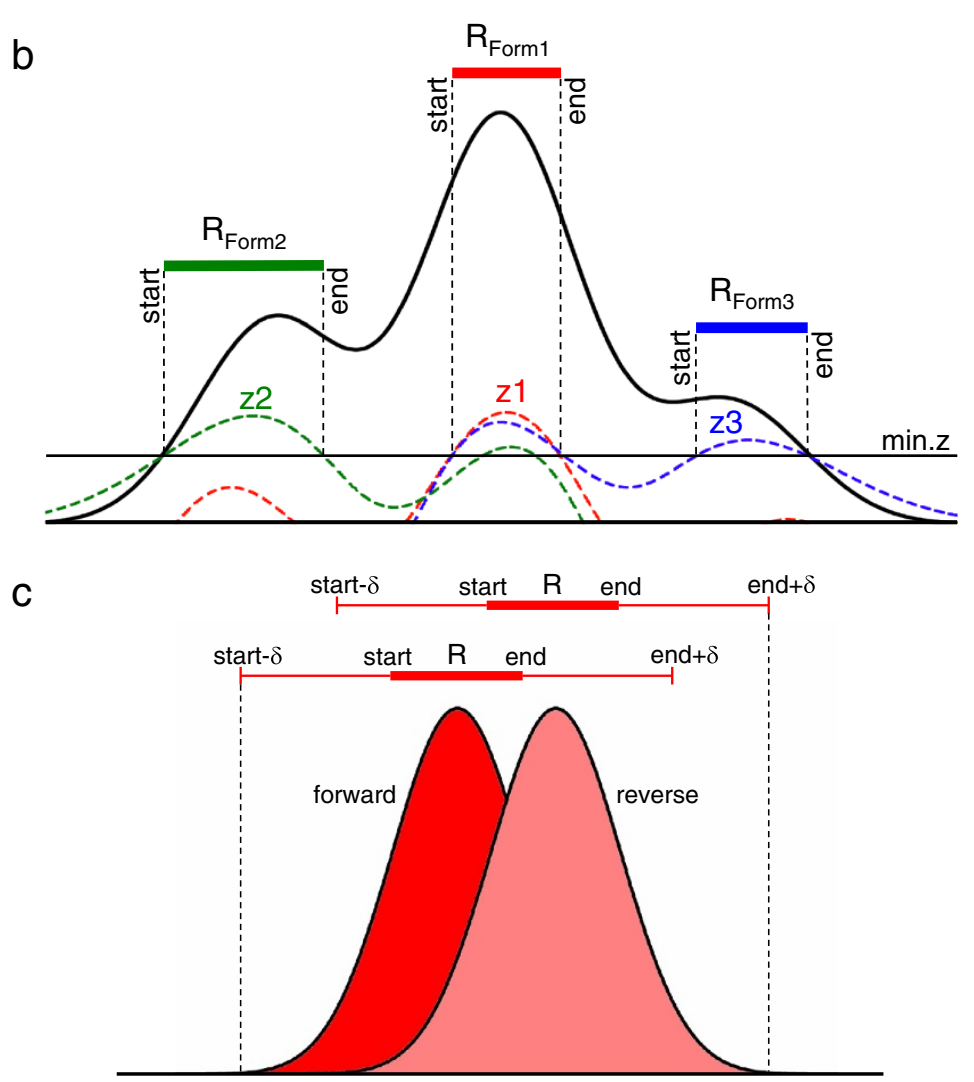

Figure 1 Triform approach to peak definition. Key aspects of the Triform algorithm. Part a) shows the definition of the three alternative peak-shape hypotheses Forms 1, 2, and 3 using very simplified peaks. Part b) shows this on a more realistic peak shape, using the min.z cut-off on the $z 1, z 2$, and $z 3$ test statistics to define the start and end of the regions $\mathrm{R}$ of each peak-like form. Part c) shows how strand lag for a peak pair on the forward (red) and reverse (pink) strand is estimated, using the $\delta$ parameter to define an extended region. Triform uses cross-correlation on this region to estimate the optimal lag, and compares this with the minimum inter-strand lag min.lag.

The necessary and sufficient conditions to detect a local peak-like form within a region $\mathrm{R}$ on one strand are:

- The number of base pairs in region $\mathrm{R}$ (peak width) exceeds a given minimum min.n.

- For every location $x$ in region $\mathrm{R}: z f(x ; \delta)>$ min.z, where $z f$ denotes one of the standard normal test functions $z 1, z 2$, and $z 3$ defined by equations (4)-(6) above (see Figure $1 \mathrm{~b}$ ), and min. $z$ is a fixed minimum upper-tail z-value cut-off for Hoel tests.

- For every location $x$ in region $\mathrm{R}: z 4(x)>\min . z$.

- For location $x_{0}$ at the center of region R: $E R\left(x_{0}\right)>$ min.er.

- If biological replicates are available, then for every replicate $k$ and every location $x$ in region $\mathrm{R}$ : $z f(x ; k, \delta)>0$ and $z 4(x ; k)>0$. 
The criteria to detect local peak-like forms on both strands are:

- For each of the three peak forms accept only those detected peak regions that overlap exactly one detected peak region on the opposite strand.

- Accept only those pairs of overlapping peak regions whose local inter-strand lag exceeds a given minimum min.lag (see Figure 1c).

- Reject any redundant Form 2 or Form 3 peaks that overlap a Form 1 peak.

- Merge any overlapping Form 2 and Form 3 peaks into Form 1 peaks.

\section{Implementation of the Triform algorithm}

The Triform method has been implemented in $\mathrm{R}$ using the IRanges package. For each detected peak region, the peak position (PEAK.LOC) is reported as the midpoint of the range, and the peak significance (PEAK.NLP) is reported as the sum of the Negative $\log 10$ (P) (NLP) values for the test statistics calculated according to equations (4)-(6). This is proportional to the Fisher's $\chi^{2}$ statistics for the combined null hypothesis that all individual null hypotheses are true. Details of the implementation can be found in Additional file 1. The Triform algorithm has been implemented in $\mathrm{R}$, and is available via http://tare.medisin.ntnu.no/triform. It has been submitted to Bioconductor for inclusion in the Bioconductor package [15].

\section{Triform outperforms other methods on a manually curated ChIP-Seq benchmark}

We evaluated the performance of Triform on the manually curated ChIP-Seq benchmark for peak-like enrichment profiles, created by Rye et al. [6] (see Figure 2). The performance of Triform was compared with that of seven other programs for ChIP-Seq peak identification: QuEST, MACS, the Meta approach by Rye et al., PICS, FindPeaks, PeakRanger, and TPic. QuEST and MACS are popular, and have both performed well in previous program evaluations $[4,5]$. Meta was the method used by Rye et al. during the original benchmark, and is a combination of outputs from the programs MACS and SISSRs [7]. PICS uses a Bayesian approach to identify binding events [16]. FindPeaks tests for local peak-like coverage distributions [9] and is the tool that is currently most similar to the Triform algorithm. PeakRanger and TPic are recent additions to the family of ChIP-Seq peak-identification programs, and have, to our knowledge, not yet been independently evaluated.

Triform clearly outperformed all these methods on peak finding, for all three TFs that were analyzed, both with respect to coverage, which is the percentage of true peaks identified, and the FDR. Most importantly, Triform recovered $80 \%$ of the peaks at a 0.05 FDR level. For NRSF and SRF, Triform kept the FDR at an acceptable 0.1 when the coverage went beyond $90 \%$. For the more challenging MAX data set, the FDR was somewhat higher when the coverage exceeded 90\%. However, given the differences in binding patterns for the three factors, especially exemplified by MAX that includes many partly overlapping peaks, Triform showed consistently good performance for all factors.

\section{Triform shows good performance on a motif enrichment benchmark}

A frequently used benchmark approach is to test genomic regions from a peak finder for binding site motifs for the TF sampled in the ChIP-Seq experiment, assuming that the best performing peak finders will identify peak regions with a high occurrence rate of relevant motifs. Here we used the approach described for example in the evaluation of PICS [16]. Output from each peak finder was sorted according to significance, and for the top $n$ peak regions a representative binding site motif for the given TF was used to scan each region for significant binding sites. Please see Methods for details. Both the fraction of regions containing binding sites, and the average distance from the estimated peak summit was estimated. Results for MAX using the MAX binding site motif from Jaspar [17] is shown in Figure 3; full results for all three TFs are found in Additional file 2: Figure S1 to S3.

The good performance of Triform on the motif enrichment benchmark confirms that the superior performance on the peak finding benchmark is consistent with a high biological relevance. Triform also seems to have the best overall performance with respect to finding the optimal peak summit. Apart from this, the difference between methods with respect to motif enrichment is relatively small, except for a couple of methods with sub-optimal performance, and the relative performance of all methods seems to vary with data set and motif model. This test is easily biased for example by the choice of motif description, the relative frequency of indirect binding of TFs, and possible lack of specificity of the antibody used in the ChIP-Seq experiment. This is therefore a more indirect test of peak finder performance than the peak profile benchmark described in the previous sub-section.

\section{Triform peaks generally show the most significant statistical association with relevant biological processes}

As an evaluation of the general quality of the peak set defined by selected methods, the highest-scoring peaks were submitted to GREAT [18] for statistical overrepresentation analysis. As the total number of peaks varied, 

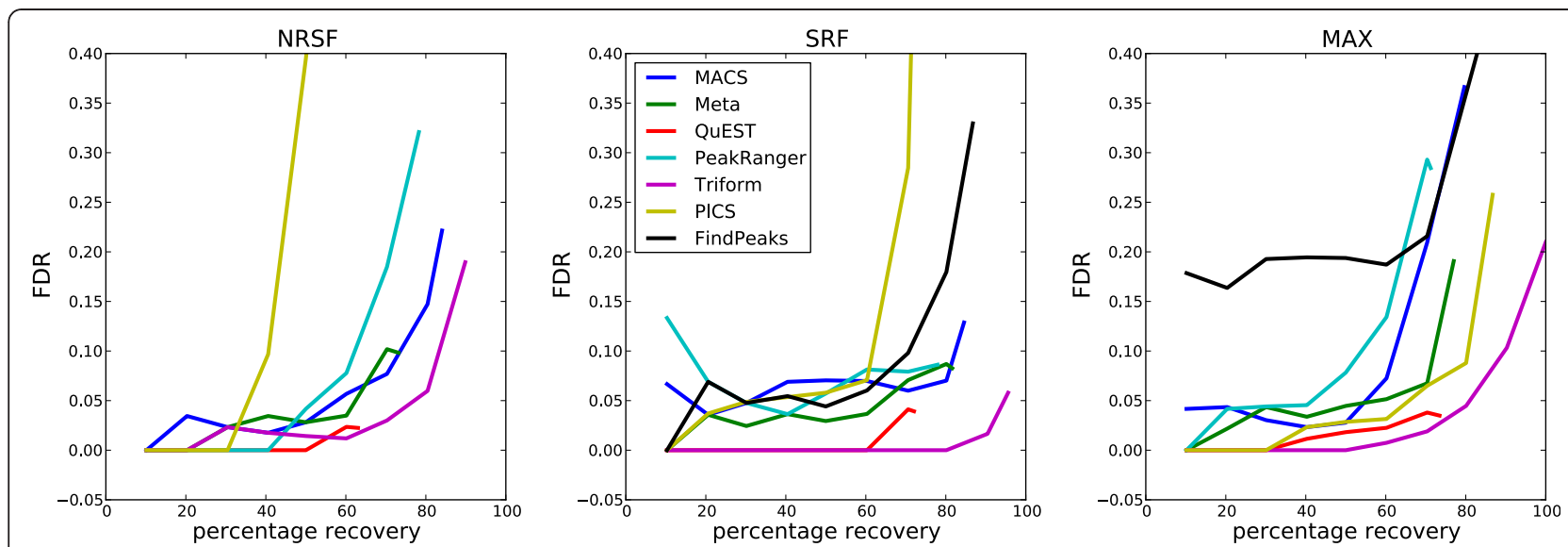

Figure 2 Peak profile benchmark results. Triform outperforms other methods on ChIP-Seq peak profile benchmarks for the TFs NRSF, SRF, and MAX. Coverage, which is the percentage of true peaks recovered in the evaluated regions, is plotted against the false discovery rate (FDR) for each method evaluated. The peak lists from each method are first sorted according to the peak scores from that program. The corresponding coverage and FDR are then calculated as the list is traversed starting at the highest scoring peak. In general, Triform has the lowest FDR for medium-to-high coverage. To emphasize the details at the lower FDR levels, the results from TPic are not shown because of the high FDRs produced by this method. The results from FindPeaks for NRSF are not shown due to the low total number of regions that were recovered (389), giving a total coverage of only $7 \%$.
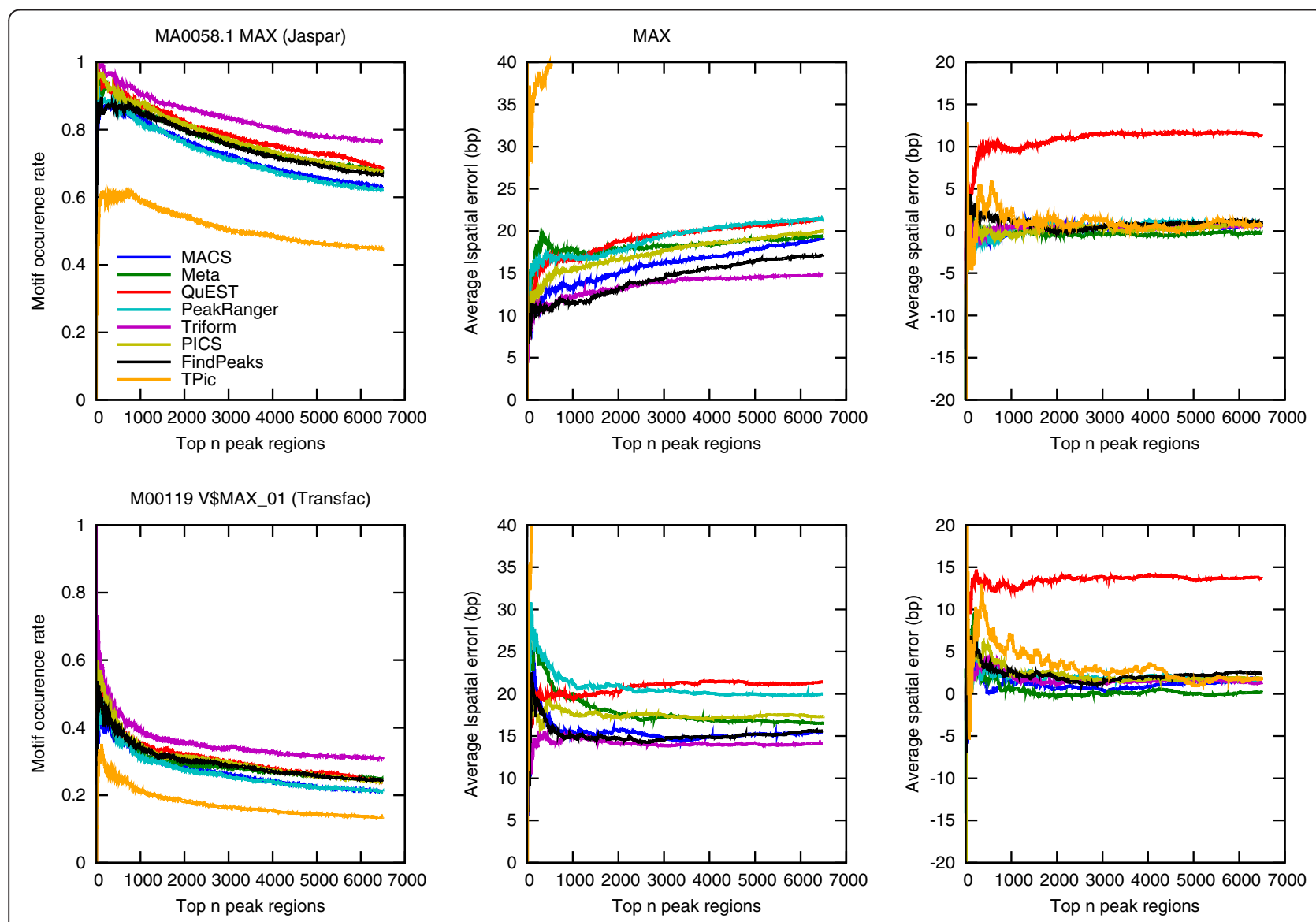

Figure 3 Motif enrichment benchmark results. Triform shows good performance on the motif enrichment benchmark. The figure shows motif occurrence rate of the Jaspar MAX motif (MA0058.1) in peak regions ( \pm 150 bp around predicted peak summit) sorted according to significance. Also shown is the average absolute and relative spatial error of the motif position relative to the predicted peak summit. If more than one significant motif was found in a given region, only the motif closest to the predicted peak summit was used for statistics. Please see Additional file 2 for full results. 
depending on both the data set and prediction method, a fixed number of peaks representing only those with highest score was used in each case. This was to make the conditions as similar as possible for the different tools. These peak lists were submitted to GREAT, which associates peaks (entered as genomic regions) with genes, and test gene annotations for statistically overrepresented terms relative to a general genomic background. Selected properties of the overrepresentation analysis were evaluated and are summarized in Table 2 . These features were ranked high by all or most of the methods, and represent in general known processes or features associated with each of the TFs.

The analysis of NRSF/REST was based on the top 3500 peaks from each method, and the peak regions showed an overlap from $94 \%$ to $64 \%$ among the different methods. GREAT associated on average 4000 genes with these peak regions. Overrepresentation analysis focused on the gene ontology (GO) feature "Neurotransmitter transport" and TF feature "REST motif" from Predicted Promoter Motifs. The highlighted GO feature is supported by previous publications $[19,20]$. For both features, Triform showed the most significant overrepresentation.
The analysis of SRF was based on 1300 regions, with an inter-method overlap between $77 \%$ and $39 \%$. GREAT associated on average 1800 genes with these peak regions. Overrepresentation analysis focused on the GO feature "Actin cytoskeleton", which is supported, for example, by the findings of Sun et al. [21], and on the TF feature "SRF motif". Here, Meta showed the most significant overrepresentation of GO features, whereas Triform was best on the TF feature. However, the difference between Meta and Triform was small and barely significant.

Finally, the analysis of MAX was based on 6500 regions, with an inter-method overlap between $75 \%$ and $49 \%$. On average, 6300 genes were associated with these peak regions. Here, the variation among the methods with respect to overrepresented features was much larger. Therefore, several features were compared: two GO features ("ncRNA processing" and "DNA duplex unwinding") and three TF features ("MYC motif" and "E2F motif" from Predicted Promoter Analysis, and "c-MYC from ChIP-chip" from Perturbation). The interaction between MAX and MYC is well documented (e.g., [22]). The interaction between MYC (and therefore probably MAX) and E2F has also been demonstrated previously

Table 2 Results of statistical overrepresentation analysis with GREAT ${ }^{\mathrm{a}}$

\begin{tabular}{|c|c|c|c|c|c|c|}
\hline Method & MACS & Meta & QuEST & PeakRanger & TPic & Triform \\
\hline \multicolumn{7}{|l|}{ NRSF } \\
\hline Peaks total (2894 common) & 9802 & 4750 & 3574 & 6123 & 37514 & 10583 \\
\hline Peaks used (1993 common) & 3500 & 3500 & 3500 & 3500 & 3500 & 3500 \\
\hline Genes & 4017 & 4017 & 4105 & 3956 & 3866 & 4033 \\
\hline GO - Neurotransmitter transport & $1.6 \times 10^{-10}$ & $6.0 \times 10^{-11}$ & $1.2 \times 10^{-10}$ & $2.5 \times 10^{-6}$ & $5.2 \times 10^{-7}$ & $9.3 \times 10^{-13}$ \\
\hline TF - REST motif & $1.1 \times 10^{-26}$ & $4.6 \times 10^{-25}$ & $5.4 \times 10^{-19}$ & $1.4 \times 10^{-25}$ & $5.6 \times 10^{-23}$ & $1.1 \times 10^{-31}$ \\
\hline \multicolumn{7}{|l|}{ SRF } \\
\hline Peaks total (608 common) & 4012 & 1553 & 1383 & 1320 & 111305 & 9555 \\
\hline Peaks used (310 common) & 1300 & 1300 & 1300 & 1300 & 1300 & 1300 \\
\hline Genes & 1930 & 1887 & 1855 & 1816 & 1565 & 1837 \\
\hline GO - Actin cytoskeleton & $1.8 \times 10^{-7}$ & $2.6 \times 10^{-9}$ & $1.2 \times 10^{-6}$ & $2.1 \times 10^{-8}$ & - & $3.8 \times 10^{-9}$ \\
\hline TF - SRF-motif & $6.0 \times 10^{-9}$ & $3.6 \times 10^{-12}$ & $2.4 \times 10^{-11}$ & $3.6 \times 10^{-12}$ & $2.4 \times 10^{-11}$ & $2.3 \times 10^{-18}$ \\
\hline \multicolumn{7}{|l|}{ MAX } \\
\hline Peaks total (3306 common) & 21866 & 10323 & 6556 & 9383 & 61021 & 15137 \\
\hline Peaks used (2014 common) & 6500 & 6500 & 6500 & 6500 & 6500 & 6500 \\
\hline Genes & 6139 & 6393 & 6626 & 6283 & 6276 & 6668 \\
\hline GO - ncRNA processing & $4.3 \times 10^{-18}$ & $5.9 \times 10^{-14}$ & $2.0 \times 10^{-18}$ & $3.9 \times 10^{-22}$ & $3.1 \times 10^{-16}$ & $1.5 \times 10^{-12}$ \\
\hline GO - DNA duplex unwinding & $9.7 \times 10^{-43}$ & $3.9 \times 10^{-14}$ & - & $1.6 \times 10^{-5}$ & $1.1 \times 10^{-25}$ & - \\
\hline TF - MYC motif & $2.5 \times 10^{-12}$ & $2.7 \times 10^{-14}$ & $5.3 \times 10^{-16}$ & - & - & $1.7 \times 10^{-17}$ \\
\hline TF - E2F1 motif & $2.7 \times 10^{-12}$ & $1.8 \times 10^{-12}$ & - & $7.3 \times 10^{-15}$ & $5.4 \times 10^{-13}$ & $2.9 \times 10^{-11}$ \\
\hline TF - c-MYC from ChIP-chip & $2.1 \times 10^{-36}$ & $2.8 \times 10^{-35}$ & $8.7 \times 10^{-48}$ & $3.9 \times 10^{-36}$ & $1.8 \times 10^{-46}$ & $7.8 \times 10^{-58}$ \\
\hline
\end{tabular}

${ }^{\mathrm{a}}$ The table shows (for each TF) the total number of peaks identified by each peak-finder (including the number of peaks common to all methods), the number of peaks used for GREAT analysis (including the number of common peaks), the number of associated genes identified by GREAT, and the binominal FDR Q-values identified by GREAT for the selected GO and TF-associated terms. The smallest Q-value for each term is shown in bold. 
[23]. The relevance of the GO properties is supported for example by [24], which discusses the role of MYC in the regulation of ncRNA expression, and by [25] on the role of MYC in replication. In this analysis, Triform showed the best performance on two of the TF features (see Table 2 for details).

Because of the somewhat inconsistent results for MAX, two additional tests were performed on this data set. First, a smaller data set was tested, using only the 3500 most significant peaks. Second, a moving window approach was used to select the peak sets, shifting the window 1000 peaks down the ranked list for each test. The rationale behind this was to test whether this data set contained a mixed signal, where the weaker peaks represented regulation of other processes (for example through a co-factor), compared with the stronger peaks. However, neither of these tests provided clarification, and in general, the significance was reduced.

In summary, overrepresentation analysis shows that the Triform method tends to identify peaks that are significantly associated with relevant biological processes.

\section{Triform peak regions facilitate the detection of co-occurring SRF/ELK1/NFY motifs in LTR/ERV1/MER57 repeats}

It has recently been shown [26] that a significant fraction of STAT1-binding sites are found in the primatespecific MER41 repeat. This illustrates the potential role of repeat regions in gene regulation [27], and makes it relevant to investigate other TFs similarly. However, analysis of repeat regions in ChIP-Seq data may be challenging. The mapping of reads from repeat regions is often not unique, potentially leading to more noisy peaks in these regions. This makes it beneficial to use a peakfinder with high sensitivity and specificity for nonoptimal peak shapes.

We used the SRF ChIP-Seq set, as this TF is known to associate with viral long terminal repeats (LTRs) [28]. We focused the analysis on 1510 Form 1 Triform peaks with PEAK.NLP $>12$. We initially used 2410 low-significance TPic SRF peaks as negative control regions, assuming that, given the high number of peaks returned by TPic, these regions were most likely to be SRF-binding-like regions without significant regulatory importance (at least in this context). However, using the 2522 least significant Triform SRF peaks (PEAK.NLP < 4) gave a similar result, and was used for the analysis shown here. We then used CisFinder for de novo motif discovery in the Triform peak regions ( $\pm 150 \mathrm{bp}$ around the peak summit), and compared these motifs with known motifs.

CisFinder found three significantly overrepresented motifs (Figure 4): an SRF-like motif known as the CArG box, an ELK1-like motif (CCGGAA), and an NFY-like motif (CCAAT). The most likely identity of these motifs

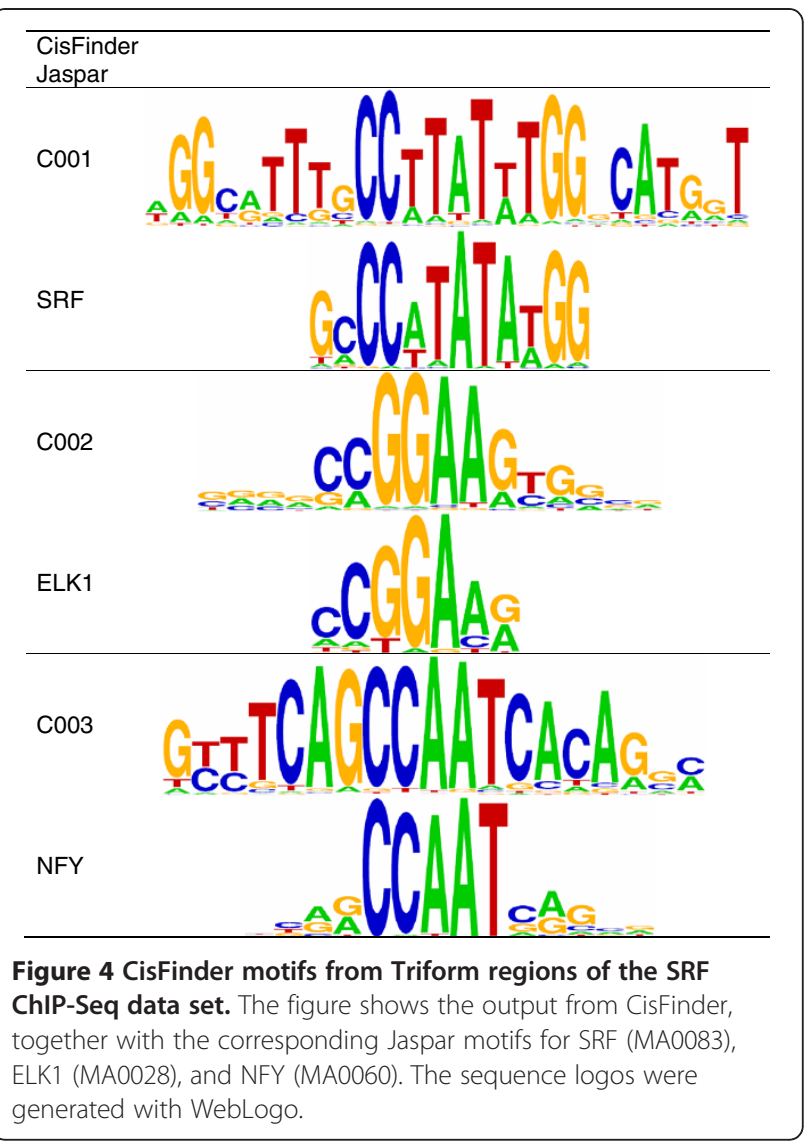

was determined by comparison of the position-specific frequency matrices against the Jaspar database using the T-Reg Comparator, identifying the most similar Jaspar motifs to be MA0083, MA0028, and MA0060, respectively. The co-occurrence of CArG box and ELK1-like motifs is well documented [29,30]. In addition, the cooccurrence of the SRF and NFY motifs has been observed previously [31-33]. The SRF/ELK1/NFY cooccurrence was strongly associated with LTRs of the medium reiteration sequence type (MER) from endogenous retrovirus (ERV) transposons (LTR/ERV1/MER57). Of the 9555 SRF regions from Triform, 124 regions overlapped with MER57 repeats (with an average overlap of $91 \%$ ), and 117 of these contained at least two significant binding sites for TFs (from the CisFinder analysis), which is highly significant ( $p=1.2 \times 10^{-65}$ according to a Fisher exact test). Submitting these MER57 regions to GREAT with a general genome-wide list of MER57 regions as background showed that the subset of repeat regions identified here co-locates with genes significantly associated with cancer (thyroid and gastric cancer, with FDR Q-values of $1.9 \times 10^{-2}$ and $3.6 \times 10^{-2}$, respectively). GREAT also indicated that this subset of MER57 regions is located in more gene-rich regions than the general 
background set, as the foreground set of MER57 regions on average was associated with 1.7 genes, compared with 0.5 genes for the full set of MER57 regions.

There is a potential risk of bias in de novo motif discovery involving multiple repeat regions, as any motif associated with repeats will be highly significant. Although the fraction of MER57 repeats is low (1.3\% of the total set), the CisFinder motif discovery was repeated with the same negative background regions, but using a positive set where all regions overlapping MER57 repeats had been removed. The final SRF-like and ELK1-like motifs were almost identical to the ones identified on the full sequence set. The NFY-like motif was also very similar, but was found at a lower frequency. This shows that the presence of MER57 repeats had only a minor effect on CisFinder motif discovery, in particular for the SRF and ELK1-like motifs.

It has long been suspected that LTRs may play a role in carcinogenesis [34]. The most likely mechanism seems to be that LTRs act as extra promoters leading to modified (possibly aberrant) expression of potential oncogenes $[35,36]$. In that respect, it is interesting that the MER57 repeats identified here co-locate with tumor-associated genes, where they may act as additional promoters. It has also recently been shown that tumor-associated microvesicles contain high levels of retrotransposon RNA transcripts [37], indicating that retroelement expression itself may play a role in carcinogenesis [38]. It is too early to say whether the subset of MER57 repeats identified here is involved in any of these processes. However, our analysis shows that using an approach for peak finding with improved sensitivity and specificity can generate interesting hypotheses for further testing.

\section{Conclusions}

It is challenging to evaluate ChIP-Seq peak-finding methods because we normally do not know the true solution to a given experiment. The intensity of a given peak reflects the frequency of interaction between the TF and the genomic region, and thus the interaction strength. It is therefore tempting to focus on the strong peaks, assuming that these represent the most important regulatory interactions. However, this may be misleading for several reasons. First, the apparent binding strength may be affected in many different ways, including posttranslational modifications and protein-protein interactions involving the relevant TF. The formation of cis-regulatory modules, including indirect binding, may also affect the efficiency of immunoprecipitation and pull-down of the relevant genomic fragments. The mapping of reads to the reference genome may also be biased, for example by differences between the sequenced genome and the reference genome used for read mapping. This means that we also have to include minor peaks in the analysis, which makes the peak finding more challenging. In addition, many protein-protein interactions lead to more complicated peak shapes, making the problem even more challenging.

Triform attempts to improve peak finding by identifying and using essential features of typical ChIP-Seq peaks, including peak shape and sequencing-induced peak shifts. This has been implemented in a rigorous model-free statistical framework, making the classification both robust and sensitive. In particular, Triform achieves greater sensitivity, specificity, and control of the FDR than other methods by utilizing the Hoel test to detect significant Poisson inhomogeneities, as could be seen in its comparison with, for example, FindPeaks.

As Triform gains performance by addressing specific properties of ChIP-Seq peaks, it could be argued that Triform may lead to model overfitting by favoring features that are important mainly in the benchmark set. However, the selected features represent completely general ChIP-Seq peak features, and the number of parameters in the Triform implementation has been reduced to a minimum. In combination with the statistical framework, we believe that this makes the algorithm more resistant to overfitting.

The excellent performance of Triform has been confirmed by the tests described here, including benchmarking, statistical overrepresentation analysis, and motif discovery for novel motifs. In all cases, the evaluation was limited by the fact that no perfect solution is available as a reference. However, in our opinion, all these tests indicate that Triform is at least as good as any of the methods it has been compared with, and in many cases is better.

However, it is important to be aware that although the Triform approach uses a quite general framework, the implementation is adapted to peak finding in ChIP-Seq experiments for TFs. It is likely that application to other types of ChIP-Seq experiments, for example for chromatin structure, will require a modified approach.

\section{Methods}

\section{Data sets}

The ChIP-Seq benchmark data set is based on sets of manually evaluated regions for three TFs: NRSF/REST, SRF, and MAX [6]. All the original ChIP-Seq tag files and results from the manual evaluations were downloaded from http://tare.medisin.ntnu.no/chipseqbenchmark/.

The full data sets for NRSF/REST, SRF, and MAX were downloaded from the ENCODE [39] repository of the UCSC Genome Browser [40] as specified in [6], and these data were used for peak finding for motif enrichment benchmark and statistical overrepresentation analysis. Identification of co-occurring peaks in repeats was based on the SRF data set. The list of 
MER57-type repeats for hg18 was downloaded from the UCSC Genome Browser.

\section{Software tools}

The following program versions were used in the evaluations: QuEST v2.4 [41], MACS v1.4.0 [8], PICS v1.0.6 [16], FindPeaks v4.0 (as part of the Vancouver Short Read Analysis Package v4.0.16) [9], PeakRanger v1.02 [42], TPic from January, 2011 [10], and the Meta approach from March, 2011 [6]. All programs were run with default parameters, and the peaks from each program were sorted according to the score given in that program. Exceptions are TPic, and to some extent FindPeaks. TPic does not return any score for its final peaks. We therefore sorted the peaks from TPic according to tag enrichment. FindPeaks returns identical score values for large sub-sets of peaks, and these sub-sets were therefore subsequently sorted according to tag enrichment, in order to rank all peaks. For all programs, including Triform, samples and replicates were pooled into a single sample before analysis. To make the peak-lengths returned by the different programs comparable, we used the peak summit with a 150-bp extension in both directions as peak regions for all programs. A region length of $300 \mathrm{bp}$ is in accordance with the resolution offered by most ChIP-Seq data.

Statistical overrepresentation analysis of predicted peak sets was performed with GREAT version 1.8.2 [18]. BEDTools [43] was used for general manipulation of the peak lists, including estimates of overlap between the lists. For motif discovery in the motif enrichment benchmark we used Patser v3e [44], reporting all motifs with score at least equal to the negative value of the samplesize adjusted information content (option -li). TF motif matrices were taken from Jaspar [17], using the default vertebrate matrices for REST, SRF and MAX. Additional matrices were taken from Transfac [45], using the matrices most similar to the Jaspar matrices according to STAMP [46], except for SRF, where the Jaspar matrix has relatively low performance (see Additional file 2: Figure S2). Here the Transfac matrix V\$SRF_01 is the one most similar to Jaspar SRF, but the alternative Transfac matrix V\$SRF_Q6 showed better performance, and was therefore preferred. For de novo motif discovery, we used CisFinder [47] with CG/AT adjustment (removing spurious repetitive GC-rich patterns) and a minimum enrichment ratio of three. The motifs were compared with the relevant Jaspar [17] entries using the T-Reg Comparator [48], and final motif logos were generated with WebLogo [49].

The Triform algorithm was implemented in $\mathrm{R}$ using the IRanges package from Bioconductor [15] The ccf function from the R statistical analysis package was used to find the lag with maximum cross-correlation between the forward and reverse coverage distributions.

\section{Additional files}

Additional file 1: Supplementary Information. Implementation of the Triform algorithm.

Additional file 2: Full results of motif enrichment benchmark test. Figure S1 - Results for NRSF. Figure S2 - Results for SRF. Figure S3 Results for MAX.

\section{Abbreviations}

bp: base pairs; ChIP-Seq: Chromatin immunoprecipitation combined with high-throughput sequencing; ERV: Endogenous retrovirus; FDR: False discovery rate; GO: Gene ontology; LTR: Long terminal repeat; MER: Medium reiteration sequence; NLP: Negative Log10 (P); TF: Transcription factor.

\section{Competing interests}

The authors declare that they have no competing interests.

\section{Authors' contributions}

KK developed the Triform method. KK and TH developed the

R-implementation. MBR performed peak profile benchmarking. FD did the motif enrichment benchmark, evaluated the Triform results and drafted the manuscript. All authors contributed to and approved the final manuscript.

\section{Acknowledgments}

We thank the FUGE Bioinformatics core facility at the Norwegian University of Science and Technology (NTNU) for technical support. The work was supported by the FUGE project of the Research Council of Norway through FUGE Bioinformatics [183438/S10], and by internal FUGE funding at NTNU.

\section{Author details}

'Division of Sensory Biophysics, Ohio State University, Columbus, OH, USA. ${ }^{2}$ Department of Cancer Research and Molecular Medicine, Norwegian University of Science and Technology (NTNU), P.O. Box 8905, NO-7491 Trondheim, Norway.

Received: 3 January 2012 Accepted: 21 June 2012

Published: 24 July 2012

\section{References}

1. Barski A, Zhao K: Genomic location analysis by ChIP-Seq. J Cell Biochem 2009, 107(1):11-18.

2. Johnson DS, Mortazavi A, Myers RM, Wold B: Genome-wide mapping of in vivo protein-DNA interactions. Science 2007, 316(5830):1497-1502.

3. Park PJ: ChIP-seq: advantages and challenges of a maturing technology. Nat Rev Genet 2009, 10(10):669-680

4. Laajala TD, Raghav S, Tuomela S, Lahesmaa R, Aittokallio T, Elo LL: A practical comparison of methods for detecting transcription factor binding sites in ChIP-seq experiments. BMC Genomics 2009, 10:618.

5. Wilbanks EG, Facciotti MT: Evaluation of algorithm performance in ChIP-seq peak detection. PLoS One 2010, 5(7):e11471.

6. Rye MB, Saetrom P, Drablos F: A manually curated ChIP-seq benchmark demonstrates room for improvement in current peak-finder programs. Nucleic Acids Res 2011, 39(4):e25.

7. Jothi R, Cuddapah S, Barski A, Cui K, Zhao K: Genome-wide identification of in vivo protein-DNA binding sites from ChIP-Seq data. Nucleic Acids Res 2008, 36(16):5221-5231.

8. Zhang Y, Liu T, Meyer CA, Eeckhoute J, Johnson DS, Bernstein BE, Nusbaum C, Myers RM, Brown M, Li W, et al: Model-based analysis of ChIP-Seq (MACS). Genome Biol 2008, 9(9):R137.

9. Fejes AP, Robertson G, Bilenky M, Varhol R, Bainbridge M, Jones SJ: FindPeaks 3.1: a tool for identifying areas of enrichment from massively parallel shortread sequencing technology. Bioinformatics 2008, 24(15):1729-1730.

10. Hower V, Evans SN, Pachter L: Shape-based peak identification for ChIP-Seq. BMC Bioinformatics 2011, 12:15.

11. Xu H, Handoko L, Wei $X$, Ye C, Sheng J, Wei CL, Lin F, Sung WK: A signalnoise model for significance analysis of ChIP-seq with negative control. Bioinformatics 2010, 26(9):1199-1204.

12. Szalkowski AM, Schmid CD: Rapid innovation in ChIP-seq peak-calling algorithms is outdistancing benchmarking efforts. Brief Bioinform 2011 12(6):626-633 
13. Kharchenko PV, Tolstorukov MY, Park PJ: Design and analysis of ChIP-seq experiments for DNA-binding proteins. Nat Biotechnol 2008, 26(12):1351-1359.

14. Hoel PG: Testing the homogeneity of Poisson frequencies. Ann Math Stat 1945, 16(4):362-368.

15. Gentleman RC, Carey VJ, Bates DM, Bolstad B, Dettling M, Dudoit S, Ellis B, Gautier L, Ge Y, Gentry J, et al: Bioconductor: open software development for computational biology and bioinformatics. Genome Biol 2004, 5(10):R80.

16. Zhang X, Robertson G, Krzywinski M, Ning K, Droit A, Jones S, Gottardo R: PICS: probabilistic inference for ChIP-seq. Biometrics 2011, 67(1):151-163.

17. Portales-Casamar E, Thongjuea S, Kwon AT, Arenillas D, Zhao X, Valen E, Yusuf D, Lenhard B, Wasserman WW, Sandelin A: JASPAR 2010: the greatly expanded open-access database of transcription factor binding profiles. Nucleic Acids Res 2010, 38:D105-D110.

18. McLean CY, Bristor D, Hiller M, Clarke SL, Schaar BT, Lowe CB, Wenger AM, Bejerano G: GREAT improves functional interpretation of cis-regulatory regions. Nat Biotechnol 2010, 28(5):495-501.

19. Palm K, Belluardo N, Metsis M, Timmusk T: Neuronal expression of zinc finger transcription factor REST/NRSF/XBR gene. J Neurosci 1998, 18(4):1280-1296.

20. Bruce AW, Donaldson IJ, Wood IC, Yerbury SA, Sadowski MI, Chapman M, Gottgens B, Buckley NJ: Genome-wide analysis of repressor element 1 silencing transcription factor/neuron-restrictive silencing factor (REST/NRSF) target genes. Proc Natl Acad Sci U S A 2004, 101(28):10458-10463.

21. Sun Q, Chen G, Streb JW, Long X, Yang Y, Stoeckert CJ Jr, Miano JM: Defining the mammalian CArGome. Genome Res 2006, 16(2):197-207.

22. Kato GJ, Lee WM, Chen LL, Dang CV: Max: functional domains and interaction with c-Myc. Genes Dev 1992, 6(1):81-92.

23. Leung JY, Ehmann GL, Giangrande PH, Nevins JR: A role for Myc in facilitating transcription activation by E2F1. Oncogene 2008, 27(30):4172-4179.

24. Kenneth NS, White RJ: Regulation by c-Myc of ncRNA expression. Curr Opin Genet Dev 2009, 19(1):38-43.

25. Robinson K, Asawachaicharn N, Galloway DA, Grandori C: c-Myc accelerates S-phase and requires WRN to avoid replication stress. PLoS One 2009, 4(6):e5951.

26. Schmid CD, Bucher P: MER41 repeat sequences contain inducible STAT1 binding sites. PLoS One 2010, 5(7):e11425.

27. Cohen CJ, Lock WM, Mager DL: Endogenous retroviral LTRs as promoters for human genes: a critical assessment. Gene 2009, 448(2):105-114.

28. Wycuff DR, Yanites HL, Marriott SJ: Identification of a functional serum response element in the HTLV-I LTR. Virology 2004, 324(2):540-553.

29. Cahill MA, Nordheim A, Janknecht R: Co-occurrence of CArG boxes and TCF sites within viral genomes. Biochem Biophys Res Commun 1994, 205(1):545-551.

30. Boros J, Donaldson IJ, O'Donnell A, Odrowaz ZA, Zeef L, Lupien M, Meyer CA, Liu XS, Brown M, Sharrocks AD: Elucidation of the ELK1 target gene network reveals a role in the coordinate regulation of core components of the gene regulation machinery. Genome Res 2009, 19(11):1963-1973.

31. Zachow KR, Conklin KF: CArG, CCAAT, and CCAAT-like protein binding sites in avian retrovirus long terminal repeat enhancers. J Virol 1992, 66(4):1959-1970.

32. Yamada K, Osawa $H$, Granner DK: Identification of proteins that interact with NF-YA. FEBS Lett 1999, 460(1):41-45.

33. Yokoyama KD, Ohler U, Wray GA: Measuring spatial preferences at fine-scale resolution identifies known and novel cis-regulatory element candidates and functional motif-pair relationships. Nucleic Acids Res 2009 37(13):e92.

34. Druker $\mathrm{R}$, Whitelaw E: Retrotransposon-derived elements in the mammalian genome: a potential source of disease. J Inherit Metab Dis 2004, 27(3):319-330.

35. Buzdin A, Kovalskaya-Alexandrova E, Gogvadze E, Sverdlov E: At least $50 \%$ of human-specific HERV-K (HML-2) long terminal repeats serve in vivo as active promoters for host nonrepetitive DNA transcription. J Virol 2006, 80(21):10752-10762.
36. Lamprecht B, Walter K, Kreher S, Kumar R, Hummel M, Lenze D, Kochert K, Bouhlel MA, Richter J, Soler E, et al: Derepression of an endogenous long terminal repeat activates the CSF1R proto-oncogene in human lymphoma. Nat Med 2010, 16(5):571-579.

37. Balaj L, Lessard R, Dai L, Cho YJ, Pomeroy SL, Breakefield XO, Skog J: Tumour microvesicles contain retrotransposon elements and amplified oncogene sequences. Nat Commun 2011, 2:180.

38. Goering W, Ribarska T, Schulz WA: Selective changes of retroelement expression in human prostate cancer. Carcinogenesis 2011 32(10):1484-1492

39. Birney E, Stamatoyannopoulos JA, Dutta A, Guigo R, Gingeras TR, Margulies EH, Weng Z, Snyder M, Dermitzakis ET, Thurman RE, et al: Identification and analysis of functional elements in $1 \%$ of the human genome by the ENCODE pilot project. Nature 2007, 447(7146):799-816.

40. Kent WJ, Sugnet CW, Furey TS, Roskin KM, Pringle TH, Zahler AM, Haussler D: The human genome browser at UCSC. Genome Res 2002, 12(6):996-1006.

41. Valouev A, Johnson DS, Sundquist A, Medina C, Anton E, Batzoglou S, Myers RM, Sidow A: Genome-wide analysis of transcription factor binding sites based on ChIP-Seq data. Nat Methods 2008, 5(9):829-834.

42. Feng X, Grossman R, Stein L: PeakRanger: a cloud-enabled peak caller for ChIP-seq data. BMC Bioinformatics 2011, 12:139.

43. Quinlan AR, Hall IM: BEDTools: a flexible suite of utilities for comparing genomic features. Bioinformatics 2010, 26(6):841-842.

44. Hertz GZ, Stormo GD: Identifying DNA and protein patterns with statistically significant alignments of multiple sequences. Bioinformatics 1999, 15(7-8):563-577.

45. Matys V, Kel-Margoulis OV, Fricke E, Liebich I, Land S, Barre-Dirrie A, Reuter I, Chekmenev D, Krull M, Hornischer K, et al: TRANSFAC and its module TRANSCompel: transcriptional gene regulation in eukaryotes. Nucleic Acids Res 2006, 34:D108-D110.

46. Mahony S, Benos PV: STAMP: a web tool for exploring DNA-binding motif similarities. Nucleic Acids Res 2007, 35:W253-W258

47. Sharov AA, Ko MS: Exhaustive search for over-represented DNA sequence motifs with CisFinder. DNA Res 2009, 16(5):261-273.

48. Roepcke S, Grossmann S, Rahmann S, Vingron M: T-Reg Comparator: an analysis tool for the comparison of position weight matrices. Nucleic Acids Res 2005, 33:W438-W441.

49. Crooks GE, Hon G, Chandonia JM, Brenner SE: WebLogo: a sequence logo generator. Genome Res 2004, 14(6):1188-1190.

doi:10.1186/1471-2105-13-176

Cite this article as: Kornacker et al:: The Triform algorithm: improved sensitivity and specificity in ChIP-Seq peak finding. BMC Bioinformatics 2012 13:176

\section{Submit your next manuscript to BioMed Central and take full advantage of:}

- Convenient online submission

- Thorough peer review

- No space constraints or color figure charges

- Immediate publication on acceptance

- Inclusion in PubMed, CAS, Scopus and Google Scholar

- Research which is freely available for redistribution 